\title{
Outcomes of surgical treatment for upper urinary tract transitional cell carcinoma: Comparison of retroperitoneoscopic and open nephroureterectomy
}

\author{
Tawatchai Taweemonkongsap*, Chaiyong Nualyong, \\ Teerapon Amornvesukit, Sunai Leewansangtong, Sittiporn Srinualnad, \\ Bansithi Chaiyaprasithi, Phichaya Sujijantararat, Anupan Tantiwong and \\ Suchai Soontrapa
}

Address: Division of Urology, Department of Surgery, Faculty of Medicine Siriraj Hospital, Mahidol University, Bangkok, Thailand

Email: Tawatchai Taweemonkongsap* - sittm@mahidol.ac.th; Chaiyong Nualyong - sicny@mahidol.ac.th; Teerapon Amornvesukit - sitav@mahidol.ac.th; Sunai Leewansangtong - sislt@mahidol.ac.th; Sittiporn Srinualnad - sisri@mahidol.ac.th; Bansithi Chaiyaprasithi - C_Bansithi@yahoo.com; Phichaya Sujijantararat - sipsj@mahidol.ac.th; Anupan Tantiwong - siatt@mahidol.ac.th; Suchai Soontrapa - sissl@mahidol.ac.th

* Corresponding author

Published: 15 January 2008

World Journal of Surgical Oncology 2008, 6:3 doi:10.1186/1477-7819-6-3

This article is available from: http://www.wjso.com/content/6/l/3

(C) 2008 Taweemonkongsap et al; licensee BioMed Central Ltd.

This is an Open Access article distributed under the terms of the Creative Commons Attribution License (http://creativecommons.org/licenses/by/2.0), which permits unrestricted use, distribution, and reproduction in any medium, provided the original work is properly cited.

Received: 10 September 2007

Accepted: 15 january 2008

\begin{abstract}
Objectives: To determine the surgical and oncologic outcomes in patients who underwent retroperitoneoscopic nephroureterectomy (RNU) in comparison to standard open nephroureterectomy (ONU) for upper urinary tract transitional cell carcinoma (TCC).

Patients and methods: From April 200I to January 2007, 60 total nephroureterectomy were performed for upper tract TCC at Siriraj Hospital. Of the 60 patients, thirty-one were treated with RNU and open bladder cuff excision, and twenty-nine with ONU. Our data were reviewed and analyzed retrospectively. The recorded data included sex, age, history of bladder cancer,
\end{abstract} type of surgery, tumor characteristics, postoperative course, disease recurrence and progression.

Results: The mean operative time was longer in the RNU group than in the ONU group $(258.8$ versus I 90.6 min; $p=0 .<001)$. On the other hand, the mean blood loss and the dose of parenteral analgesia (morphine sulphate) were lower in the RNU group (289.3 versus $313.7 \mathrm{ml}$ and 2.05 versus $6.72 \mathrm{mg} ; \mathrm{p}=0.868$ and $\mathrm{p}=0.018$, respectively). There were two complications in each group. No significant difference in $p$ stage and grade in both-groups $(p=0.95 \mathrm{I}, \mathrm{p}=0.077)$. One patient with RNU had lymph node involvement, three in ONU. Mean follow up was 26.4 months (range 3-72) for RNU and 27.9 months (range 3-63) for ONU. No port metastasis occurred during follow up in RNU group. Tumor recurrence developed in II patients (bladder recurrence in 9 patients, local recurrence in 2 patients) in the RNU group and 14 patients (bladder recurrence in 13 patients, local recurrence in I patient) in the ONU group. No significant difference was detected in the tumor recurrence rate between the two procedures $(\mathrm{p}=0.2716)$. Distant metastases developed in 3 patients $(9.7 \%)$ after RNU and 2 patients $(6.9 \%)$ after ONU. The 2 year disease specific survival rate after RNU and ONU was $86.3 \%$ and $92.5 \%$, respectively $(p=0.8227)$.

Conclusion: Retroperitoneoscopic nephroureterectomy is less invasive than open surgery and is an oncological feasible operation. Thus, the results of our study supported the continued development of laparoscopic technique in the management of upper tract TCC. 


\section{Background}

The standard surgical procedure to treat upper urinary tract transitional cell carcinoma (TCC) is open nephroureterectomy (ONU) with bladder cuff excision. However, the morbidity of open surgery (e.g. severe pain and prolonged convalescence) is inevitable. In 1991, Clayman firstly described the technique of laparoscopic nephroureterectomy (LNU), which was soon replicated by various authors worldwide [1]. Recently, LNU through the transperitoneal or retroperitoneal approach has been used to treat upper urinary tract TCC, with reduced morbidity [2]. Although the many other benefits of LNU are clear, the application of these techniques to the treatment of cancer raises issues relating to oncologic safety. Up to date, most studies have shown the oncologic outcomes of LNU comparable to ONU groups $[3,4]$. However, few reports with adequate follow up in upper tract TCC patients after retroperitoneoscopic nephroureterectomy (RNU) have been published [5-7]. To determine whether the surgical and oncologic outcomes of RNU is at least equivalent to that of ONU, we present our 7 years experience of RNU with open bladder cuff excision, compared with patients after ONU, in upper urinary tract TCC treatment.

\section{Patients and methods}

From April 2001 to January 2007, 60 patients underwent total nephroureterectomy with bladder cuff excision for upper tract TCC at Faculty of Medicine Siriraj Hospital. According to the decision of the surgeon's preference, 31 patients were treated with RNU, and 29 patients with ONU. In all patients, the surgery was performed completely extraperitoneal with open bladder cuff technique. Upper tract TCC was diagnosed by intravenous urography, retrograde pyelography, computed tomography of the abdomen, magnetic resonance imaging, and ureteroscopy with or without biopsy. Preoperative cystoscopy and radiologic examinations were performed to rule out metastasis and concomitant bladder cancer.

LNU was performed using the retroperitoneal approach. The patient was placed in a lateral position. After a retroperitoneal working space had been created, the pneumoretroperitoneum was maintained with carbon dioxide gas at $10 \mathrm{mmHg}$. Three or four trocars were inserted in the usual manner. The posterior peritoneum was mobilized medially so that dissection of Gerota's fascia and the renal pedicle could be fully performed. After the lymphatic channels around the renal pedicle were excised to expose the renal artery, this artery was isolated, clipped and divided. The renal vein was mobilized and secured with clips. Caudally, the fatty tissue around the ureter was divided at the level of the iliac vessels crossing. Finally the kidney was completely mobilized. Lymphadenectomy was performed at surgeon's discretion. The patient posi- tion was then changed to supine. An approximately $7 \mathrm{~cm}$ long Gibson's incision was made, and the distal ureter with a bladder cuff specimen was removed en bloc without opening the urinary tract. If the cancer was located in the mid or distal ureter, lymphadenectomy was consecutively performed around the lesion.

The standard ONU was performed using a flank incision. The distal ureter management was performed as standard technique. All patients with concomitant bladder tumor were underwent transurethral resection concomitantly.

All patients with proven nodal disease were counseled for adjuvant therapy. Patients have a follow-up cystoscopy every 3 months in the first 2 years, every 6 months in the following 3 years and annually after 5 years.

We retrospectively reviewed our database and extracted data on the following variables: sex, age at diagnosis, history of previous bladder cancer, type of surgery, complications, tumor characteristics, postoperative course, disease recurrence and disease progression.

The comparison between the two groups was carried out using the Mann-Whitney $U$ test and Fisher's exact test. Time to recurrence was evaluated from the date of surgery. Recurrence free survival was defined as the interval from surgery to the first tumor recurrence, the detection of distant metastases or the end of the study. Survivals were analyzed by the Kaplan-Meier method. To assess the effect of type of surgery on time to recurrence after adjusting for the effects of pathological stage and grading, a Cox's proportional hazard model was fitted. For all statistical tests, $\mathrm{P}<0.05$ was considered to indicate a significant difference.

\section{Results}

The characteristics of the patients who underwent RNU and ONU are shown in Table 1. There was no significant difference in mean age $(\mathrm{p}=0.353)$, operative side $(\mathrm{p}=$ $0.796)$, tumor location ( $\mathrm{p}=0.233)$, and concomitant or history of bladder cancer $(\mathrm{p}=0.599)$.

A comparison of the perioperative parameters between the two groups is shown in Table 2. No significant differences were founded in blood transfusion, mean time to first diet, length of indwelling urethral catheter, and hospital stay. The mean operative time was significant longer in the RNU group $(\mathrm{p}=<0.001)$. However, although not to a significant extent, the mean blood loss tended to be less in the RNU group ( 289 vs. $313 \mathrm{ml}$ ).

Additionally, the dose of parenteral analgesia was significantly reduced in RNU group $(\mathrm{p}=0.018)$. Complications developed in 2 patients of each group. In the RNU group, 
Table I: Patient characteristics

\begin{tabular}{|c|c|c|c|}
\hline \multirow[t]{2}{*}{ Variable } & \multicolumn{3}{|c|}{ Number (\%) or Mean (Min-Max) } \\
\hline & RNU (N = 3I) & ONU (N = 29) & P Value \\
\hline Age, years & $63.8(26-79)$ & $66.8(39-88)$ & 0.353 \\
\hline \multicolumn{4}{|l|}{ Sex } \\
\hline Male & II (35.5) & $22(75.9)$ & \\
\hline Female & $20(64.5)$ & $7(24.1)$ & \\
\hline \multicolumn{4}{|l|}{ Side } \\
\hline Left & $18(58.1)$ & $15(5 \mid .7)$ & 0.796 \\
\hline Right & $13(41.9)$ & $14(48.3)$ & \\
\hline \multicolumn{4}{|l|}{ Tumor location } \\
\hline Renal pelvis & $14(45.2)$ & $10(34.5)$ & 0.233 \\
\hline Ureter & $13(41.9)$ & $10(34.5)$ & \\
\hline Multifocal & $4(12.9)$ & $9(3 \mathrm{I})$ & \\
\hline Concomitant or history of bladder cancer & II (35.5) & $13(44.8)$ & 0.599 \\
\hline
\end{tabular}

one patient had ischemic heart disease which required coronary angiography. Another patient had postoperative urinary tract infection and required parenteral antibiotic with prolonged hospital stay. In the ONU group, one patient had postoperative bleeding which required open surgery to stop bleeding. Another patient had an urinoma at perivesical space and required surgical drainage.

The oncologic results are shown in Table 3. There were no statistical difference in tumor stage and grade in both groups ( $\mathrm{p}=0.951$ and $\mathrm{p}=0.077$, respectively). Lymphadenectomy was performed in 20 patients $(64.5 \%)$ with RNU and 9 patients (31.0\%) with ONU groups. One patient in each group was found to have a single lymph node micrometastasis. Both patients were managed conservatively due to refuse chemotherapy and further follow-up to 30, 31 months respectively showed no evidence of disease recurrence. Another two patients in ONU group had multiple lymph node metastasis. One patient developed bone metastasis after 8 months despite adjuvant systemic chemotherapy. Another patient, with large persisting lymph node at resection site, died of to tumor progression after 7 months. It was noted that this patient had no adjuvant therapy due to poor performance status.

There was no port site metastasis occurred during follow up in RNU group. Bladder cancer recurrence occurred in 9 patients $(29 \%)$ in the RNU group and 13 patients $(44.8 \%)$ in the ONU group. No statistically significant difference was observed $(P=0.285)$. Local recurrence developed in 2 patients in the RNU group and 1 patient in the ONU group, in 2 of whom distant metastases in the lung and bone were detected simultaneously. All three patients had a negative surgical margin on histopathological examination. The metastasis rate was $9.7 \%(3 / 31)$ after RNU and $6.9 \%(2 / 29)$ after ONU $(\mathrm{p}=1.00)$. The median

Table 2: Surgical results

\begin{tabular}{|c|c|c|c|}
\hline \multirow[t]{2}{*}{ Variable } & \multicolumn{3}{|c|}{ Mean (Min-Max) or Number (\%) } \\
\hline & RNU (N = 3I) & ONU (N = 29) & P Value \\
\hline Operative time (min) & $258.87(90-425)$ & $190.69(105-360)$ & $<0.001 *$ \\
\hline Blood loss (ml) & $289.35(100-800)$ & $313.79(50-800)$ & 0.868 \\
\hline Blood transfusion & $6(19.3)$ & $7(24.1)$ & 0.758 \\
\hline Time to first diet (days) & $1.13(1-2)$ & $1.10(1-2)$ & 1.000 \\
\hline $\begin{array}{l}\text { Time to remove of urethral } \\
\text { catheter (days) }\end{array}$ & $6.81(2-16)$ & $6.24(1-11)$ & 0.727 \\
\hline Hospital stay (days) & $9.32(6-20)$ & $8.69(5-13)$ & 0.890 \\
\hline \multicolumn{4}{|l|}{ Parenteral analgesia } \\
\hline Morphine sulphate $(\mathrm{mg})$ & $2.05(0-10)$ & $6.72(0-35)$ & $0.018^{*}$ \\
\hline \multicolumn{4}{|l|}{ Complication } \\
\hline Ischemic heart disease & 1 & 0 & \\
\hline Urinary tract infection & i & 0 & \\
\hline Re-explor (bleeding) & 0 & 1 & \\
\hline Urinoma & 0 & 1 & \\
\hline
\end{tabular}


Table 3: Oncologic results

\begin{tabular}{lcc}
\hline & Variable & Number (\%) or Mean (Min-Max) \\
\cline { 2 - 3 } & RNU (N = 3I) & ONU (N = 29) \\
\hline Pathologic stages & & \\
TI & $16(51.6)$ & $13(44.8)$ \\
T2 & $10(32.3)$ & $12(41.4)$ \\
T3 & $4(12.9)$ & $4(13.8)$ \\
T4 & $1(3.2)$ & $0(0.0)$ \\
Grade & & $10(34.5)$ \\
Low & $18(58.1)$ & $19(65.5)$ \\
High & $13(41.9)$ & $9(31.0)$ \\
Node & $20(64.5)$ & $6(66.7)$ \\
Negative & $19(95)$ & $3(33.3)$ \\
Positive & $1(5)$ & $14(48.2)$ \\
Recurrence & $11(35.4)$ & $13(44.8)$ \\
Bladder & $9(29.0)$ & $1(3.4)$ \\
Local & $2(6.4)$ & $2(6.9)$ \\
Metastasis & $3(9.7)$ & $27.9(3-63)$ \\
Follow up time (month) & $26.4(3-72)$ & $92.5 \%$ \\
2 yr. disease specific survival & $86.3 \%$ & $83.3 \%$ \\
2 yr. overall survival & $86.3 \%$ & 0.077 \\
\hline
\end{tabular}

time to metastasis was 12 months (range 6-14) and 14 months (range 8-20) in the RNU and ONU groups, respectively. For the RNU group, three patients died of distant metastasis (two in the liver, one in the lung) and one patient died of cardiac disease during the follow up period. For the ONU group, two patients died of disease progression (one in the lung, one in the lymph node) and two patients died from other causes unrelated to tumor. The median time to recurrence was 40 months (range 371 ) and 23 months (range 3-63) in the RNU and ONU groups, respectively. The prognostic factors studied by multivariate analysis given in Table 4. Analysis results revealed that even though ONU seemed to have a higher risk of recurrence than $\mathrm{RNU}(\mathrm{HR}=1.50,95 \% \mathrm{CI}=0.67$, $3.35)$ there was no statistical difference $(\mathrm{p}=0.323)$. There was also no significant effect of stage (stage 2 : HR $=1.15$, $\mathrm{p}=0.776$; stage 3 : $\mathrm{HR}=2.58, \mathrm{p}=0.144$ ) and grade $($ High: $\mathrm{HR}=1.21, \mathrm{p}=0.701$ ) on recurrence. For recurrence free survival analysis, we found no statistically significant difference between the two procedures ( $p=0.2716)$ (Fig. 1A). Additionally, we found no statistically significant difference in recurrence free survival curves between the two procedures in terms of $\mathrm{p}$ stage and grade (Fig. 1B-E). The mean follow up time of the RNU group and the ONU group was 26.4 months (range 3-72) and 27.9 months (range 3-63) respectively. No significant difference was found between the two procedures with regard to disease specific and overall survival (Fig. 2A, B). The 2 years disease specific survival rate was $86.3 \%$ in the RNU group and $92.5 \%$ in the open group $(P=0.8227)$. The corresponding 2 years overall survival rate was $86.3 \%$ and $83.3 \%(P=0.8628)$.

\section{Discussion}

Laparoscopic nephroureterectomy was developed in an effort to reduce the morbidity of the surgical management. Indeed, several investigators have recently suggested their benefit for patient recovery with disease control comparable to that of traditional open surgery [2$4]$. The mean oral diet day, urethral catheter time, and hospital stay were equivalent in the both groups in our series. However, the operative time was longer in the laparoscopic groups. On the other hand, the blood loss and the dosage of analgesia were lower after laparoscopic nephroureterectomy. In a literature review of 1365 nephroureterectomy patients, Rassweiler et al. reported the operative time ( 277 vs. $220 \mathrm{~min}$ ) and the blood loss (241 vs. $463 \mathrm{ml}$.) comparing between the laparoscopic series and open series [2]. These findings correspond to our results and support the effectiveness of laparoscopic procedure compared with the standard open procedure.

Laparoscopic nephroureterectomy can be performed via a transperitoneal or retroperitoneal access. We used the retroperitoneal approach. Although the operating space is smaller and a more skilled technique is required than with the transperitoneal approach, the advantage of retroperitoneal approach in avoiding intraabdominal injury and tumor spillage into intraabdominal cavity are our consideration. Rouprêt et al. reported the complications of colonic injury after transperitoneal LNU [4]. We found no complication of intraabdominal injury and two minor complications after retroperitoneal LNU in our series. These finding confirmed the benefit of retroperitoneal approach and a feasible technique for LNU. Additionally, 

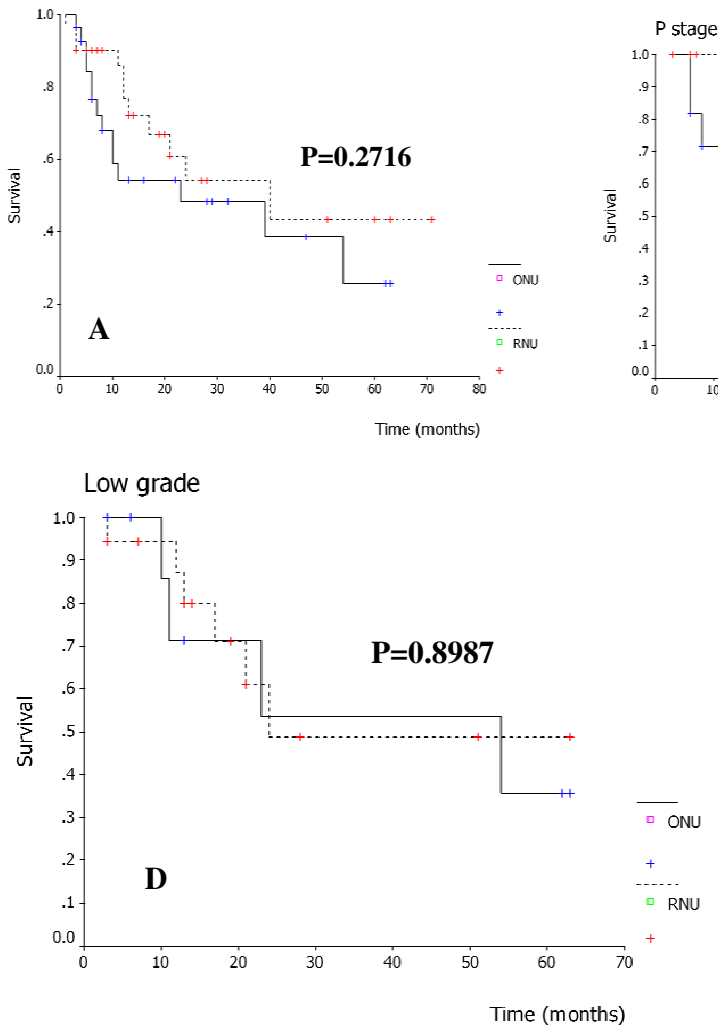
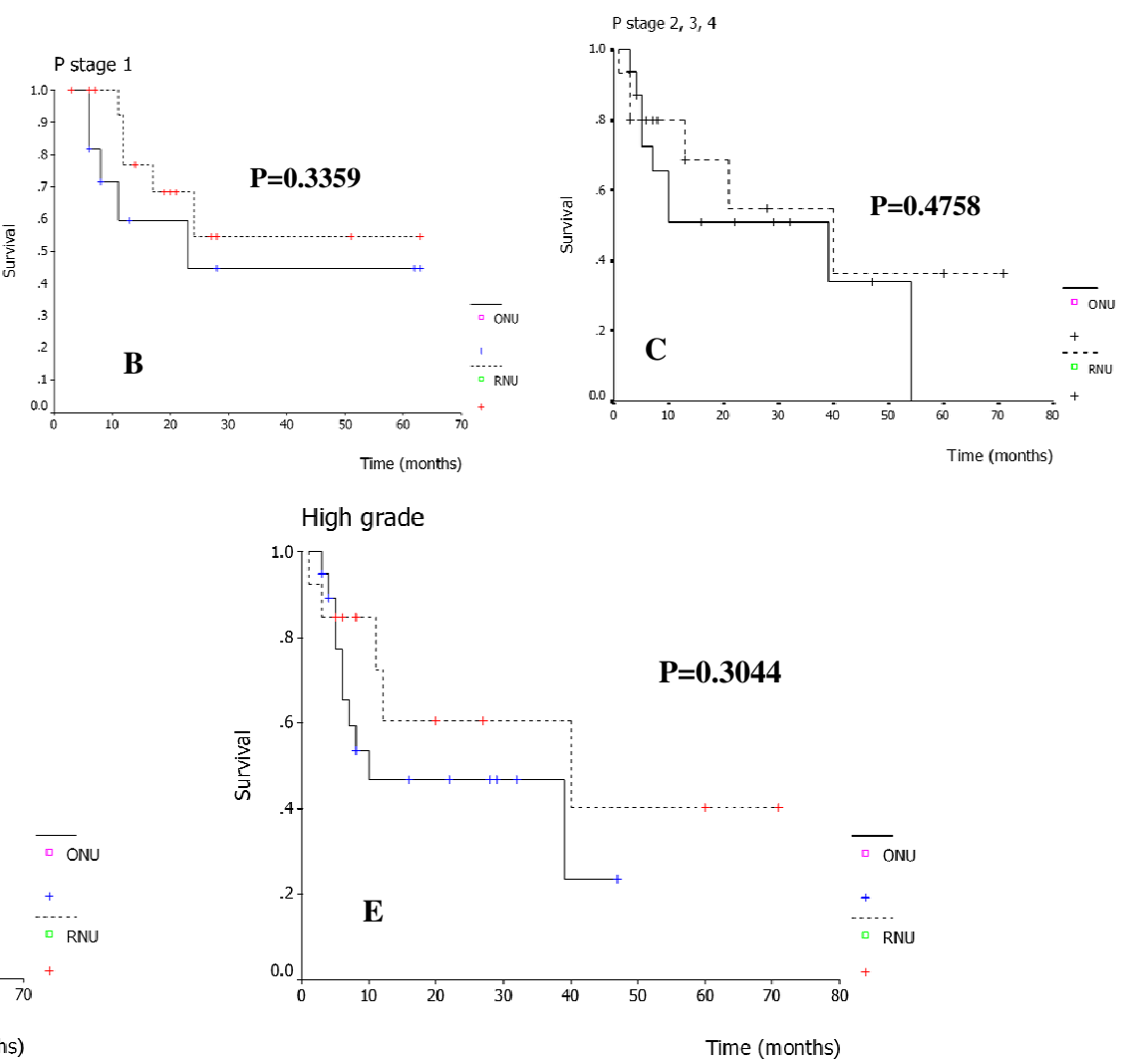

Figure I

Recurrence free survival according to surgical procedure $(A)$, stage $(B, C)$, grade $(D, E)$.

the technique of ureterectomy and bladder cuff excision has not been standardized yet. A number of minimal invasive approaches to the distal ureter such as endoscopic stripping or pluck-off techniques have been reported [811]. However, these endoscopic techniques have a greater risk of local recurrence and stone formation in the staple lines [12]. We prefer open distal ureterectomy and bladder cuff excision. This method avoids the risk of urinary leakage and allows for intact specimen removal. We believed this will not adversely affect patient's recovery compared with the endoscopic approach. Furthermore, there are no contraindications such as ureteral tumors or periureteral fibrosis due to previous surgery, irradiation or inflammatory pelvic disease [13]. The worldwide reported bladder recurrence rate was $9-48 \%$ with different methods for controlling the bladder cuff $[2,14,15]$. In our series, the bladder recurrence rate (29\%) after RNU was within the reported range. In addition, the problem of port site metastasis in laparoscopic procedure is important. Rassweiler et al. reported that six port site metastasis in $377(1.6 \%)$ analyzed patients following laparoscopy were recognized [2]. Recently, Schatteman et al. reported another three cases of port metastasis after laparoscopy
[16]. In most cases, extraction of the specimen was performed without an organ or with a torn organ bag. In our series, no case of port site metastasis was observed during the follow up period. We routinely avoid the use of harmonic scalpel for tissue dissection which might be an origin of tumor cell spreading as previously described [17] and we retrieved the intact specimen via the open wound.

The indication for laparoscopic nephroureterectomy in upper tract TCC is not yet well defined. Although most authors still recommended that high stage and grade tumors should be contraindications to LNU $[2,3,5]$. Recently in 2007, Muntener et al. reported oncologic outcome after LNU with a median follow up time of 74 months and supported the LNU as the standard of care for high grade or high stage upper tract TCC [18]. In our series, we found no statistically significant difference in recurrence free survival curve between both procedures in terms of tumor grade and stage (Fig. 1B-E). However, we believe that the indication tend to increase as surgical skill developed in laparoscopic treatment and we could have identified additional candidates with high grade or high 

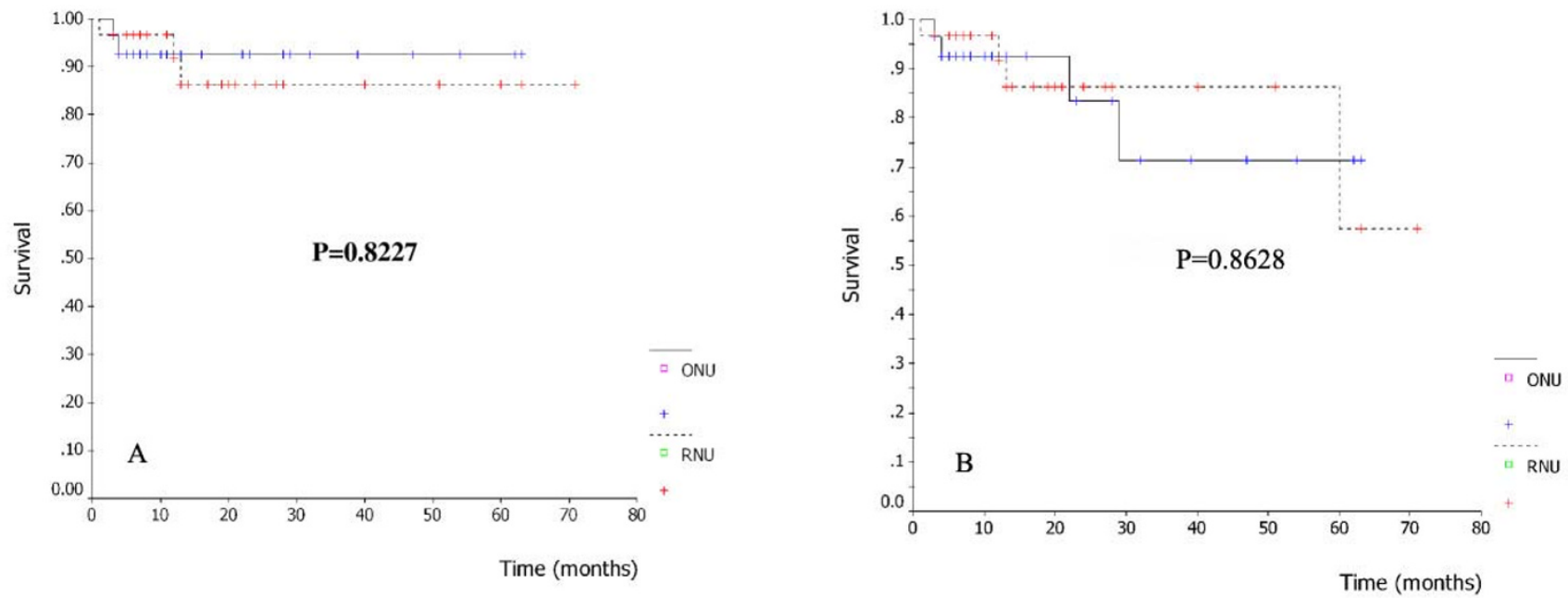

Figure 2

Disease specific survival (A), overall survival (B) according to surgical procedure.

stage tumor for LNU if accurate staging with preoperative imaging and biopsy had been done.

McNeill et al. reported favorable long term outcomes after LNU compared with ONU; however, information on nodal status was available in only $4 \%$ of cases [19]. Klinger et al. found micrometastasis in $14.3 \%$ ( 2 of 14) of clinical No patients and advised to perform lymphadenectomy routinely for staging purpose [17]. In our series, lymphadenectomy was performed in $48.3 \%$ $(29 / 60)$ of cases. We had no definitive criteria for choosing the surgical procedure, including the indication for lymphadenectomy, which might affect the results of treatment. We found micrometastasis in 2 patients and these patients are still alive until the last follow up time. However, the prospective randomized study is needed to support the benefit and efficacy of routine laparoscopic regional lymphadenectomy.

In 2000 Gill et al. reported retroperitoneoscopic nephroureterectomy with bladder cuff excision through a transvesical approach and at a mean follow up of 11 months the cancer specific survival rate was $97 \%$ in the LNU group

Table 4: Results of Cox's regression

\begin{tabular}{lcccc}
\hline & b & Hazard ratio (HR) & 95\% Cl of HR & p-value \\
\hline ONU & 0.406 & 1.50 & $0.67,3.35$ & 0.323 \\
Stage 2 & 0.139 & 1.15 & $0.44,3.00$ & 0.776 \\
Stage 3 & 0.947 & 2.58 & $0.72,9.18$ & 0.144 \\
High grade & 0.189 & 1.21 & $0.46,3.17$ & 0.701 \\
\hline
\end{tabular}

[20]. Hsueh et al. reported Hand assisted RNU with open bladder cuff excision compare to ONU [7]. The study showed no significant difference in terms of the disease specific and overall survival rate between the two groups. In 2007, Manabe et al. reported oncologic outcome of LNU with the same surgical approach as in our study. The study showed the 2 years disease specific survival rate were similar in both groups ( 85.2 vs $87 \%$ ) [21]. The worldwide reported disease survival was $72-95 \%$ with different methods for LNU and distal ureter management $[16,17,22]$. In the present series shows a 2 years disease specific survival of $86.3 \%$ which is comparable to literature data. No significant difference in disease specific and overall survival curve were found between both procedures. These results confirmed the oncologic safety of retroperitoneoscopic nephrectomy compared with the standard ONU.

\section{Conclusion}

The retroperitoneoscopic nephroureterectomy with open bladder cuff excision seems to be a safe alternative treatment for upper urinary tract TCC and offers the advantages of laparoscopic procedure. From the oncologic stand point, it is not associated with an increased risk of tumor recurrence compared with the standard open neprhoureterectomy. Because of limitation in retrospective study, thus a true prospective and continued evaluation of longer follow up data are needed before RNU should become the new standard of care for the upper tract TCC.

\section{Competing interests}

The author(s) declare that they have no competing interests. 


\section{Authors' contributions}

TT conceived and participated in the study performed statistical analysis interpreted the data and prepared the draft manuscript. TA and BC helped in interpretation of data and preparation of the manuscript; $\mathrm{CN}$, SL, SIS participated in acquisition of data and preparation of manuscript; PS, AT and SUS helped designing the study and manuscript preparation. All authors read and approved final manuscript for publication.

\section{References}

I. Clayman RV, Kavoussi LR, Figenshau RS, Chandhoke PS, Albala DM: Laparoscopic nephroureterectomy: initial clinical case report. J Laparoendosc Surg 1991, I:343-349.

2. Rassweiler JJ, Schulze M, Marrero R, Frede T, Palou Redorta J, Bassi $\mathrm{P}$ : Laparoscopic nephroureterectomy for upper urinary tract transitional cell carcinoma: is it better than open surgery? Eur Urol 2004, 46:690-697.

3. El Fettouh HA, Rassweiler JJ, Schulze M, Salomon L, Allan J, Ramakumar S, Jarrett T, Abbou CC, Tolley DA, Kavoussi LR, Gill IS: Laparoscopic radical nephroureterectomy: results of an international multicenter study. Eur Urol 2002, 42:447-452.

4. Rouprêt M, Hupertan V, Sanderson KM, Harmon JD, Cathelineau X, Barret E, Vallancien G, Rozet F: Oncologic control after open or laparoscopic nephroureterectomy for upper urinary tract transitional cell carcinoma: a single center experience. Urology 2007, 69:656-66I.

5. Tsujihata M, Nonomura N, Tsujimura A, Yoshimura K, Miyagawa $Y$, Okuyama A: Laparoscopic nephroureterectomy for upper tract transitional cell carcinoma: comparison of laparoscopic and open surgery. Eur Urol 2006, 49:332-336.

6. Hattori $R$, Yoshino $Y$, Gotoh $M$, Katoh $M$, Kamihira $O$, Ono $Y$ : Laparoscopic nephroureterectomy for transitional cell carcinoma of renal pelvis and ureter: Nagoya experience. Urology 2006, 67:70I-705.

7. Hsueh TY, Huang YH, Chiu AW, Huan SK, Lee YH: Survival analysis in patients with upper urinary tract transitional cell carcinoma: a comparison between open and hand-assisted laparoscopic nephroureterectomy. BJU Int 2007, 99:632-636.

8. Roth $S$, van Ahlen $H$, Semjonow A, Hertle $L$ : Modified uretera stripping as an alternative to open surgical ureterectomy. J Urol 1996, I55: I568-157|

9. Angulo JC, Hontoria J, Sanchez-Chapado M: One-incision nephroureterectomy endoscopically assisted by transurethral ureteral stripping. Urology 1998, 52:203-207.

10. Palou J, Caparros J, Orsola A, Xavier B, Vicente J: Transurethra resection of the intramural ureter as the first step of nephroureterectomy. J Urol 1995, 154:43-44.

II. Gill IS, Soble J], Miller SD, Sung GT: A novel technique for management of the en bloc bladder cuff and distal ureter during laparoscopic nephroureterectomy. I Urol 1999, I 6 I:430-444.

12. Saika T, Nishiguchi J, Tsushima T, Nasu Y, Nagai A, Miyaji Y, Maki Y, Akaeda T, Saegusa M, Kumon H, Okayama Urogenital Cancer Collaborating Group (OUCCG): Comparative study of ureteral stripping versus open ureterectomy for nephroureterectomy in patients with transitional carcinoma of the renal pelvis. Urology 2004, 63:848-852.

13. Laguna MP, de la Rosette J): The endoscopic approach to the distal ureter in nephroureterectomy for upper urinary tract tumor. J Urol 200I, 166:2017-2022.

14. Jarrett TW, Chan DY, Cadeddu JA, Kavoussi LR: Laparoscopic nephroureterectomy for the treatment of transitional cel carcinoma of the upper urinary tract. Urology 2001, 57:448-453.

15. Kawauchi A, Fujito A, Ukimura $O$, Yoneda K, Mizutani $Y$, Miki T: Hand assisted retroperitoneoscopic nephroureterectomy: comparison with the open procedure. I Urol 2003 , I 69:890-894.

16. Schatteman P, Chatzopoulos C, Assenmacher C, De Visscher L, Jorion JL, Blaze V, Van Cleynenbreugel B, Billiet I, Van der Eecken H, Bollens R, Mottrie A: Laparoscopic nephroureterectomy for upper urinary tract transitional cell carcinoma: results of a
Belgian retrospective multicentre survey. Eur Urol 2007, 51:1633-1638.

17. Klingler HC, Lodde M, Pycha A, Remzi M, Janetschek G, Marberger $M$ : Modified laparoscopic nephroureterectomy for treatment of upper urinary tract transitional cell cancer is not associated with an increased risk of tumour recurrence. Eur Urol 2003, 44:442-447.

18. Muntener M, Nielsen ME, Romero FR, Schaeffer EM, Allaf ME, Brito FA, Pavlovich CP, Kavoussi LR, Jarrett TW: Long-term oncologic outcome after laparoscopic radical nephroureterectomy for upper tract transitional cell carcinoma. Eur Urol 2007, 51:1639-1644.

19. McNeill SA, Chrisofos M, Tolley DA: The long-term outcome after laparoscopic nephroureterectomy: a comparison with open nephroureterectomy. BJU Int 2000, 86:619-623.

20. Gill IS, Sung GT, Hobart MG, Savage SJ, Meraney AM, Schweizer DK, Klein EA, Novick AC: Laparoscopic radical nephroureterectomy for upper tract transitional cell carcinoma: the Cleveland Clinic experience. J Urol 2000, I 64: I 5 I 3-22.

21. Manabe D, Saika T, Ebara S, Uehara S, Nagai A, Fujita R, Irie S, Yamada D, Tsushima T, Nasu Y, Kumon H, Okayama Urological Research Group, Okayama, Japan: Comparative study of oncologic outcome of laparoscopic nephroureterectomy and standard nephroureterectomy for upper urinary tract transitional cell carcinoma. Urology 2007, 69:457-6I.

22. Bariol SV, Stewart GD, Mc Neill SA, Tolley DA: Oncologic contro following laparoscopic nephroureterectomy: 7 year outcome. J Urol 2004, I 72: 1805-1808.
Publish with Bio Med Central and every scientist can read your work free of charge

"BioMed Central will be the most significant development for disseminating the results of biomedical research in our lifetime. "

Sir Paul Nurse, Cancer Research UK

Your research papers will be:

- available free of charge to the entire biomedical community

- peer reviewed and published immediately upon acceptance

- cited in PubMed and archived on PubMed Central

- yours - you keep the copyright 